\title{
On the Univalence Criterion of a General Integral Operator
}

\author{
Daniel Breaz ${ }^{1}$ and H. Özlem Güney ${ }^{2}$ \\ ${ }^{1}$ Department of Mathematics, "1 Decembrie 1918" University, Alba Iulia 510009, Romania \\ ${ }^{2}$ Department of Mathematics, Faculty of Science and Arts, University of Dicle, Diyarbakir 21280, Turkey
}

Correspondence should be addressed to Daniel Breaz, dbreaz@uab.ro

Received 12 March 2008; Accepted 16 May 2008

Recommended by Paolo Ricci

In this paper we considered an general integral operator and three classes of univalent functions for which the second order derivative is equal to zero. By imposing supplimentary conditions for these functions we proved some univalent conditions for the considered general operator. Also some interesting particullar results are presented.

Copyright $\odot 2008$ D. Breaz and H. Özlem Güney. This is an open access article distributed under the Creative Commons Attribution License, which permits unrestricted use, distribution, and reproduction in any medium, provided the original work is properly cited.

\section{Introduction}

Let unit disk and let $\mathcal{A}$ denote the class of functions $f$ of the form

$$
f(z)=z+a_{2} z^{2}+a_{3} z^{3}+\cdots \quad(z \in \mathcal{U})
$$

which are analytic in the open disk $\mathcal{U}$ and satisfy the conditions $f(0)=f^{\prime}(0)-1=0$. Consider $\mathcal{S}=\{f \in \mathscr{A}: f$ are univalent functions in $\mathcal{U}\}$.

Let $\mathcal{A}_{2}$ be the subclass of $\mathcal{A}$ consisting of functions of the form

$$
f(z)=z+\sum_{k=3}^{\infty} a_{k} z^{k} .
$$

Let $\tau$ be the univalent subclass of $\mathcal{A}$ which satisfies

$$
\left|\frac{z^{2} f^{\prime}(z)}{(f(z))^{2}}-1\right|<1 \quad(z \in \mathcal{U}) .
$$


Let $\tau_{2}$ be the subclass of $\tau$ for which $f^{\prime \prime}(0)=0$. Let $\tau_{2, \mu}$ be the subclass of $\tau_{2}$ consisting of functions of the form (1.2) which satisfy

$$
\left|\frac{z^{2} f^{\prime}(z)}{(f(z))^{2}}-1\right| \leq \mu \quad(z \in \mathcal{U})
$$

for some $\mu(0<\mu \leq 1)$, and let us denote $\tau_{2,1} \equiv \tau_{2}$. Furthermore, for some real $p$ with $0<p \leq 2$ we define a subclass $\mathcal{S}(p)$ of $\mathcal{A}$ consisting of all functions $f(z)$ which satisfy

$$
\left|\left(\frac{z}{f(z)}\right)^{\prime \prime}\right| \leq p \quad(z \in \mathcal{U})
$$

In [1], Singh has shown that if $f(z) \in \mathcal{S}(p)$, then $f(z)$ satisfies

$$
\left|\frac{z^{2} f^{\prime}(z)}{(f(z))^{2}}-1\right| \leq p|z|^{2} \quad(z \in \mathcal{U}) .
$$

Ahlfors [2] and Becker [3] had obtained the following univalence criterion.

Theorem 1.1. Let $c$ be a complex number, $|c| \leq 1, c \neq-1$. If $f(z)=z+a_{2} z^{2}+\cdots$ is a regular function in $u$ and

$$
\left.|c| z\right|^{2}+\left(1-|z|^{2}\right) \frac{z f^{\prime \prime}(z)}{f^{\prime}(z)} \mid \leq 1
$$

for all $z \in \mathcal{U}$, then the function $f$ is regular and univalent in $\mathcal{U}$.

In [4], Pescar had obtained the following theorem.

Theorem 1.2 (see [4]). Let $\beta$ be a complex number, $\operatorname{Re} \beta>0, c$ a complex number, $|c| \leq 1, c \neq-1$, and $h(z)=z+a_{2} z^{2}+\cdots$ a regular function in $\mathcal{u}$. If

$$
\left.|c| z\right|^{2 \beta}+\left(1-|z|^{2 \beta}\right) \frac{z h^{\prime \prime}(z)}{\beta h^{\prime}(z)} \mid \leq 1
$$

for all $z \in \mathcal{U}$, then the function

$$
F_{\beta}(z)=\left[\beta \int_{0}^{z} t^{\beta-1} h^{\prime}(t) d t\right]^{1 / \beta}=z+\cdots
$$

is regular and univalent in $\boldsymbol{u}$.

Lemma 1.3 (the general Schwarz lemma [5]). Let the function $f(z)$ be regular in the disk $\varkappa_{R}=$ $\{z \in \mathbb{C} ;|z|<R\}$, with $|f(z)|<M$ for fixed $M$. If $f(z)$ has one zero with multiplicity order bigger than $m$ for $z=0$, then

$$
|f(z)| \leq \frac{M}{R^{m}}|z|^{m}, \quad z \in \mathcal{U}_{R}
$$

The equality can hold only if $f(z)=e^{i \theta}\left(M / R^{m}\right) z^{m}$, where $\theta$ is constant. 
D. Breaz and H. Özlem Güney

In [6], Seenivasagan and Breaz consider for $f_{i} \in \mathcal{A}_{2}(i=1,2, \ldots, n)$ and $\alpha_{1}, \alpha_{2}, \ldots, \alpha_{n}, \beta \in$ $\mathbb{C}$, the integral operator

$$
F_{\alpha_{1}, \alpha_{2}, \ldots, \alpha_{n}, \beta}(z)=\left\{\beta \int_{0}^{z} t^{\beta-1} \prod_{i=1}^{n}\left(\frac{f_{i}(t)}{t}\right)^{1 / \alpha_{i}} d t\right\}^{1 / \beta}
$$

When $\alpha_{i}=\alpha$ for all $i=1,2, \ldots, n, F_{\alpha_{1}, \alpha_{2}, \ldots, \alpha_{n}, \beta}(z)$ becomes the integral operator $F_{\alpha, \beta}(z)$ considered in [7].

\section{Main results}

Theorem 2.1. Let $M \geq 1$, let the functions $f_{i} \in \mathcal{S}(p)$, for $i \in\{1, \ldots, n\}$, satisfy the condition (1.6), let $\alpha_{i}, \beta$ be complex numbers, let $\operatorname{Re} \beta \geq \sum_{i=1}^{n}\left(((1+p) M+1) /\left|\alpha_{i}\right|\right)$, and let $c$ be a complex number. If

$$
\begin{gathered}
|c| \leq 1-\frac{1}{\operatorname{Re} \beta} \sum_{i=1}^{n} \frac{(1+p) M+1}{\left|\alpha_{i}\right|}, \\
\left|f_{i}(z)\right| \leq M
\end{gathered}
$$

for all $z \in \mathcal{U}$, then the functions $F_{\alpha_{1}, \alpha_{2}, \ldots, \alpha_{n}, \beta}$ defined in (1.11) are in the class $\mathcal{S}$.

Proof. Define a function

$$
h(z)=\int_{0}^{z} \prod_{i=1}^{n}\left(\frac{f_{i}(t)}{t}\right)^{1 / \alpha_{i}} d t
$$

then we have $h(0)=h^{\prime}(0)-1=0$. Also, a simple computation yields

$$
\begin{aligned}
h^{\prime}(z) & =\prod_{i=1}^{n}\left(\frac{f_{i}(z)}{z}\right)^{1 / \alpha_{i}} \\
\frac{z h^{\prime \prime}(z)}{h^{\prime}(z)} & =\sum_{i=1}^{n} \frac{1}{\alpha_{i}}\left(\frac{z f_{i}^{\prime}(z)}{f_{i}(z)}-1\right) .
\end{aligned}
$$

From (2.3), we have

$$
\left|\frac{z h^{\prime \prime}(z)}{h^{\prime}(z)}\right| \leq \sum_{i=1}^{n} \frac{1}{\left|\alpha_{i}\right|}\left(\left|\frac{z f_{i}^{\prime}(z)}{f_{i}(z)}\right|+1\right)=\sum_{i=1}^{n} \frac{1}{\left|\alpha_{i}\right|}\left(\left|\frac{z^{2} f_{i}^{\prime}(z)}{f_{i}^{2}(z)}\right|\left|\frac{f_{i}(z)}{z}\right|+1\right) .
$$

From the hypothesis, we have $\left|f_{i}(z)\right| \leq M(z \in \mathcal{U}, i=1,2, \ldots, n)$, then by the general Schwarz lemma, we obtain that

$$
\left|f_{i}(z)\right| \leq M|z| \quad(z \in \mathcal{U}, i=1,2, \ldots, n)
$$


We apply this result in inequality (2.4), then we obtain

$$
\begin{aligned}
\left|\frac{z h^{\prime \prime}(z)}{h^{\prime}(z)}\right| & \leq \sum_{i=1}^{n} \frac{1}{\left|\alpha_{i}\right|}\left(\left|\frac{z^{2} f_{i}^{\prime}(z)}{\left(f_{i}(z)\right)^{2}}\right| M+1\right) \\
& \leq \sum_{i=1}^{n} \frac{1}{\left|\alpha_{i}\right|}\left(\left|\frac{z^{2} f_{i}^{\prime}(z)}{\left(f_{i}(z)\right)^{2}}-1\right| M+M+1\right) \\
& =\sum_{i=1}^{n} \frac{1}{\left|\alpha_{i}\right|}\left(p M|z|^{2}+M+1\right) \\
& <\sum_{i=1}^{n} \frac{(1+p) M+1}{\left|\alpha_{i}\right|} .
\end{aligned}
$$

We have

$$
\begin{aligned}
\left.|c| z\right|^{2 \beta}+\left(1-|z|^{2 \beta}\right) \frac{z h^{\prime \prime}(z)}{\beta h^{\prime}(z)} \mid & =\left.|c| z\right|^{2 \beta}+\left(1-|z|^{2 \beta}\right) \frac{1}{\beta} \sum_{i=1}^{n} \frac{1}{\alpha_{i}}\left(\frac{z f_{i}^{\prime}(z)}{f_{i}(z)}-1\right) \mid \\
& \leq|c|+\frac{1}{|\beta|} \cdot \sum_{i=1}^{n} \frac{1}{\left|\alpha_{i}\right|}\left(\left|\frac{z^{2} f_{i}^{\prime}(z)}{f_{i}^{2}(z)}\right| \cdot \frac{\left|f_{i}(z)\right|}{|z|}+1\right) .
\end{aligned}
$$

We obtain

$$
\begin{aligned}
\left.|c| z\right|^{2 \beta}+\left(1-|z|^{2 \beta}\right) \frac{z h^{\prime \prime}(z)}{\beta h^{\prime}(z)} \mid & <|c|+\frac{1}{|\beta|} \sum_{i=1}^{n} \frac{(1+p) M+1}{\left|\alpha_{i}\right|} \\
& \leq|c|+\frac{1}{\operatorname{Re} \beta} \sum_{i=1}^{n} \frac{(1+p) M+1}{\left|\alpha_{i}\right|} .
\end{aligned}
$$

So, from (2.1) we have

$$
\left.|c| z\right|^{2 \beta}+\left(1-|z|^{2 \beta}\right) \frac{z h^{\prime \prime}(z)}{\beta h^{\prime}(z)} \mid \leq 1
$$

Applying Theorem 1.2, we obtain that $F_{\alpha_{1}, \alpha_{2}, \ldots, \alpha_{n}, \beta}$ is univalent.

Theorem 2.2. Let $M \geq 1$, let the functions $f_{i} \in \mathcal{S}(p)$, for $i \in\{1, \ldots, n\}$, satisfy the condition (1.6), let $\alpha, \beta$ be complex numbers, let $\operatorname{Re} \beta \geq n((1+p) M+1) /|\alpha|$, and let $c$ be a complex number.

If

$$
\begin{gathered}
|c| \leq 1-\frac{1}{\operatorname{Re} \beta} \frac{n((p+1) M+1)}{|\alpha|}, \\
\left|f_{i}(z)\right| \leq M
\end{gathered}
$$

for all $z \in \mathcal{u}$, then the function

$$
F_{\alpha, \beta}(z)=\left\{\beta \int_{0}^{z} t^{\beta-1} \prod_{i=1}^{n}\left(\frac{f_{i}(t)}{t}\right)^{1 / \alpha} d t\right\}^{1 / \beta}
$$

is in the class $\mathcal{S}$. 
Proof. In Theorem 2.1, we consider $\alpha_{1}=\alpha_{2}=\cdots=\alpha_{n}=\alpha$.

Corollary 2.3. Let the functions $f_{i} \in \mathcal{S}(p)$, for $i \in\{1, \ldots, n\}$, satisfy the condition (1.6), let $\alpha_{i}, \beta$ be complex numbers, let $\operatorname{Re} \beta \geq \sum_{i=1}^{n}\left((p+2) /\left|\alpha_{i}\right|\right)$, and let $c$ be a complex number.

If

$$
\begin{gathered}
|c| \leq 1-\frac{1}{\operatorname{Re} \beta} \sum_{i=1}^{n} \frac{p+2}{\left|\alpha_{i}\right|} \\
\left|f_{i}(z)\right| \leq 1
\end{gathered}
$$

for all $z \in \mathcal{U}$, then the function $F_{\alpha_{1}, \alpha_{2}, \ldots, \alpha_{n}, \beta}$ defined in (1.11) is in the class $\mathcal{S}$.

Proof. In Theorem 2.1, we consider $M=1$.

Corollary 2.4. Let $M \geq 1$, let the function $f \in \mathcal{S}(p)$ satisfy the condition (1.6), let $\alpha, \beta$ be complex numbers, let $\operatorname{Re} \beta \geq((1+p) M+1) /|\alpha|$, and let $c$ be a complex number.

If

$$
\begin{gathered}
|c| \leq 1-\frac{1}{\operatorname{Re} \beta} \frac{(1+p) M+1}{|\alpha|}, \\
|f(z)| \leq M
\end{gathered}
$$

for all $z \in \mathcal{u}$, then the function

$$
G_{\alpha, \beta}(z)=\left\{\beta \int_{0}^{z} t^{\beta-1}\left(\frac{f(t)}{t}\right)^{1 / \alpha} d t\right\}^{1 / \beta}
$$

is in the class $\mathcal{S}$.

Proof. In Theorem 2.1, we consider $n=1$.

Corollary 2.5. Let the function $f \in \mathcal{S}(p)$ satisfy the condition (1.6), let $\alpha, \beta$ be complex numbers, let $\operatorname{Re} \beta \geq(p+2) /|\alpha|$, and let $c$ be a complex number.

If

$$
\begin{gathered}
|c| \leq 1-\frac{1}{\operatorname{Re} \beta} \frac{p+2}{|\alpha|}, \\
|f(z)| \leq 1
\end{gathered}
$$

for all $z \in \boldsymbol{u}$, then the function

$$
G_{\alpha, \beta}(z)=\left\{\beta \int_{0}^{z} t^{\beta-1}\left(\frac{f(t)}{t}\right)^{1 / \alpha} d t\right\}^{1 / \beta}
$$

is in the class $\mathcal{S}$.

Proof. In Corollary 2.4, we consider $M=1$. 
Theorem 2.6. Let $M \geq 1$, let the functions $f_{i} \in \tau_{2, \mu_{i}}$, for $i \in\{1, \ldots, n\}$, satisfy the condition (1.4), let $\alpha_{i}, \beta$ be complex numbers, let $\operatorname{Re} \beta \geq \sum_{i=1}^{n}\left(\left(\left(1+\mu_{i}\right) M+1\right) /\left|\alpha_{i}\right|\right)$, and let $c$ be a complex number.

If

$$
\begin{gathered}
|c| \leq 1-\frac{1}{\operatorname{Re} \beta} \sum_{i=1}^{n} \frac{\left(1+\mu_{i}\right) M+1}{\left|\alpha_{i}\right|}, \\
\left|f_{i}(z)\right| \leq M
\end{gathered}
$$

for all $z \in \mathcal{U}$, then the function $F_{\alpha_{1}, \alpha_{2}, \ldots, \alpha_{n}, \beta}$ defined in (1.11) is in the class $\mathcal{S}$.

Proof. Define a function

$$
h(z)=\int_{0}^{z} \prod_{i=1}^{n}\left(\frac{f_{i}(t)}{t}\right)^{1 / \alpha_{i}} d t
$$

then we have $h(0)=h^{\prime}(0)-1=0$. Also a simple computation yields

$$
\begin{aligned}
h^{\prime}(z) & =\prod_{i=1}^{n}\left(\frac{f_{i}(z)}{z}\right)^{1 / \alpha_{i}}, \\
\frac{z h^{\prime \prime}(z)}{h^{\prime}(z)} & =\sum_{i=1}^{n} \frac{1}{\alpha_{i}}\left(\frac{z f_{i}^{\prime}(z)}{f_{i}(z)}-1\right) .
\end{aligned}
$$

From (2.19), we have

$$
\left|\frac{z h^{\prime \prime}(z)}{h^{\prime}(z)}\right| \leq \sum_{i=1}^{n} \frac{1}{\left|\alpha_{i}\right|}\left(\left|\frac{z f_{i}^{\prime}(z)}{f_{i}(z)}\right|+1\right)=\sum_{i=1}^{n} \frac{1}{\left|\alpha_{i}\right|}\left(\left|\frac{z^{2} f_{i}^{\prime}(z)}{f_{i}^{2}(z)}\right|\left|\frac{f_{i}(z)}{z}\right|+1\right) .
$$

From the hypothesis, we have $\left|f_{i}(z)\right| \leq M(z \in \mathcal{U}, i=1,2, \ldots, n)$, then by the general Schwarz lemma, we obtain that

$$
\left|f_{i}(z)\right| \leq M|z| \quad(z \in \mathcal{U}, i=1,2, \ldots, n)
$$

From (2.20), we obtain

$$
\begin{aligned}
\left|\frac{z h^{\prime \prime}(z)}{h^{\prime}(z)}\right| & \leq \sum_{i=1}^{n} \frac{1}{\left|\alpha_{i}\right|}\left(\left|\frac{z^{2} f_{i}^{\prime}(z)}{\left(f_{i}(z)\right)^{2}}\right| M+1\right) \\
& \leq \sum_{i=1}^{n} \frac{1}{\left|\alpha_{i}\right|}\left(\left|\frac{z^{2} f_{i}^{\prime}(z)}{\left(f_{i}(z)\right)^{2}}-1\right| M+M+1\right) \\
& \leq \sum_{i=1}^{n} \frac{1}{\left|\alpha_{i}\right|}\left(\mu_{i} M+M+1\right) \\
& =\sum_{i=1}^{n} \frac{\left(1+\mu_{i}\right) M+1}{\left|\alpha_{i}\right|} .
\end{aligned}
$$


We have

$$
\begin{aligned}
\left.|c| z\right|^{2 \beta}+\left(1-|z|^{2 \beta}\right) \frac{z h^{\prime \prime}(z)}{\beta h^{\prime}(z)} \mid & =\left.|c| z\right|^{2 \beta}+\left(1-|z|^{2 \beta}\right) \frac{1}{\beta} \sum_{i=1}^{n} \frac{1}{\alpha_{i}}\left(\frac{z f_{i}^{\prime}(z)}{f_{i}(z)}-1\right) \mid \\
& \leq|c|+\frac{1}{|\beta|} \cdot \sum_{i=1}^{n} \frac{1}{\left|\alpha_{i}\right|}\left(\left|\frac{z^{2} f_{i}^{\prime}(z)}{f_{i}^{2}(z)}\right| \cdot \frac{\left|f_{i}(z)\right|}{|z|}+1\right) .
\end{aligned}
$$

We obtain

$$
\begin{aligned}
\left.|c| z\right|^{2 \beta}+\left(1-|z|^{2 \beta}\right) \frac{z h^{\prime \prime}(z)}{\beta h^{\prime}(z)} \mid & \leq|c|+\frac{1}{|\beta|} \sum_{i=1}^{n} \frac{\left(1+\mu_{i}\right) M+1}{\left|\alpha_{i}\right|} \\
& \leq|c|+\frac{1}{\operatorname{Re} \beta} \sum_{i=1}^{n} \frac{\left(1+\mu_{i}\right) M+1}{\left|\alpha_{i}\right|} .
\end{aligned}
$$

So, from (2.17) we have

$$
\left.|c| z\right|^{2 \beta}+\left(1-|z|^{2 \beta}\right) \frac{z h^{\prime \prime}(z)}{\beta h^{\prime}(z)} \mid \leq 1 .
$$

Applying Theorem 1.2, we obtain that $F_{\alpha_{1}, \alpha_{2}, \ldots, \alpha_{n}, \beta}$ is univalent.

Corollary 2.7. Let $M \geq 1$, let the functions $f_{i} \in \tau_{2, \mu_{i}}$, for $i \in\{1, \ldots, n\}$, satisfy the condition (1.4), let $\alpha, \beta$ be complex numbers, let $\operatorname{Re} \beta \geq n\left(\left(1+\mu_{i}\right) M+1\right) /|\alpha|$, and let $c$ be a complex number.

If

$$
\begin{gathered}
|c| \leq 1-\frac{1}{\operatorname{Re} \beta} \frac{n\left(\left(1+\mu_{i}\right) M+1\right)}{|\alpha|}, \\
\left|f_{i}(z)\right| \leq M
\end{gathered}
$$

for all $z \in \mathcal{U}$, then the function

$$
F_{\alpha, \beta}(z)=\left\{\beta \int_{0}^{z} t^{\beta-1} \prod_{i=1}^{n}\left(\frac{f_{i}(t)}{t}\right)^{1 / \alpha} d t\right\}^{1 / \beta}
$$

is in the class $\mathcal{S}$.

Proof. In Theorem 2.6, we consider $\alpha_{1}=\alpha_{2}=\cdots=\alpha_{n}=\alpha$.

Corollary 2.8. Let the functions $f_{i} \in \tau_{2, \mu_{i}}$, for $i \in\{1, \ldots, n\}$, satisfy the condition (1.4), let $\alpha_{i}, \beta$ be complex numbers, let $\operatorname{Re} \beta \geq \sum_{i=1}^{n}\left(\left(\mu_{i}+2\right) /\left|\alpha_{i}\right|\right)$, and let $c$ be a complex number.

If

$$
\begin{gathered}
|c| \leq 1-\frac{1}{\operatorname{Re} \beta} \sum_{i=1}^{n} \frac{\mu_{i}+2}{\left|\alpha_{i}\right|}, \\
\left|f_{i}(z)\right| \leq 1
\end{gathered}
$$

for all $z \in \mathcal{U}$, then the function $F_{\alpha_{1}, \alpha_{2}, \ldots, \alpha_{n}, \beta}$ defined in (1.11) is in the class $\mathcal{S}$. 
Proof. In Theorem 2.6, we consider $M=1$.

Corollary 2.9. Let $M \geq 1$, let the function $f \in \tau_{2, \mu}$ satisfy the condition (1.4), let $\alpha, \beta$ be complex numbers, let $\operatorname{Re} \beta \geq((1+\mu) M+1) /|\alpha|$, and let $c$ be a complex number.

If

$$
\begin{gathered}
|c| \leq 1-\frac{1}{\operatorname{Re} \beta} \frac{(1+\mu) M+1}{|\alpha|}, \\
|f(z)| \leq M
\end{gathered}
$$

for all $z \in \mathcal{u}$, then the function

$$
G_{\alpha, \beta}(z)=\left\{\beta \int_{0}^{z} t^{\beta-1}\left(\frac{f(t)}{t}\right)^{1 / \alpha} d t\right\}^{1 / \beta}
$$

is in the class $\mathcal{S}$.

Proof. In Theorem 2.6, we consider $n=1$.

Corollary 2.10. Let the function $f \in \tau_{2, \mu}$ satisfy the condition (1.4), let $\alpha, \beta$ be complex numbers, let $\operatorname{Re} \beta \geq(\mu+2) /|\alpha|$, and let $c$ be a complex number.

If

$$
\begin{gathered}
|c| \leq 1-\frac{1}{\operatorname{Re} \beta} \frac{\mu+2}{|\alpha|}, \\
|f(z)| \leq 1
\end{gathered}
$$

for all $z \in \mathcal{u}$, then the function

$$
G_{\alpha, \beta}(z)=\left\{\beta \int_{0}^{z} t^{\beta-1}\left(\frac{f(t)}{t}\right)^{1 / \alpha} d t\right\}^{1 / \beta}
$$

is in the class $\mathcal{S}$.

Proof. In Corollary 2.9, we consider $M=1$.

\section{Acknowledgment}

D. Breaz was supported by the GAR 20/2007.

\section{References}

[1] V. Singh, "On a class of univalent functions," International Journal of Mathematics and Mathematical Sciences, vol. 23, no. 12, pp. 855-857, 2000.

[2] L. V. Ahlfors, "Sufficient conditions for quasiconformal extension," Annals of Mathematics Studies, vol. 79, pp. 23-29, 1973.

[3] J. Becker, "Löwnersche Differentialgleichung und Schlichtheitskriterien," Mathematische Annalen, vol. 202, no. 4, pp. 321-335, 1973.

[4] V. Pescar, "A new generalization of Ahlfors's and Becker's criterion of univalence," Bulletin of the Malaysian Mathematical Society. Second Series, vol. 19, no. 2, pp. 53-54, 1996.

[5] Z. Nehari, Conformal Mapping, Dover, New York, NY, USA, 1975.

[6] N. Seenivasagan and D. Breaz, "Certain sufficient conditions for univalence," General Mathematics, vol. 15, no. 4, pp. 7-15, 2007.

[7] D. Breaz and N. Breaz, "The univalent conditions for an integral operator on the classes $S_{p}$ and $T_{2}$," Journal of Approximation Theory and Applications, vol. 1, no. 2, pp. 93-98, 2005. 